\title{
Disegnare per Osservare, Capire, Raccontare. Conversazione con Agostino Sotgia
}

\section{Martina Revello Lami}

Leiden University

"C'est à force d'observations, de réflexion que l'on trouve. Ainsi, piochons, piochons continuellement"

(Claude Monet)

\section{Introduzione}

Agostino Sotgia (Roma, 1990) è dottorando in archeologia preistorica presso la "Sapienza - Università di Roma" e la "RUG - University of Groningen”. Si occupa prevalentemente di Landscape Archaeology con particolare attenzione alla ricostruzione dei paesaggi agropastorali antichi in Etruria Meridionale a cavallo del I millennio a.C. Precedentemente si è interessato alla cultura materiale con una serie di studi sulle fornaci e le aree produttive per il vasellame ceramico in Italia peninsulare tra Età del bronzo e Età arcaica. All'attività di ricerca ha affiancato anche quella di sorveglianza archeologica nei cantieri per la creazione dell'infrastrutture della fibra ottica e del Gas. Collabora, inoltre, come disegnatore di reperti archeologici presso numerosi gruppi di ricerca e singoli ricercatori.

Quando "non impugna" la matita per motivi archeologici disegna quello che gli passa per la testa e quello che si trova attorno. Recentemente ha disegnato la mascotte dell'associazione sportiva Atletico San Lorenzo ed illustrato il libro "Come un grande Sogno" (Momo Edizioni, 2020) che ne racconta la storia.

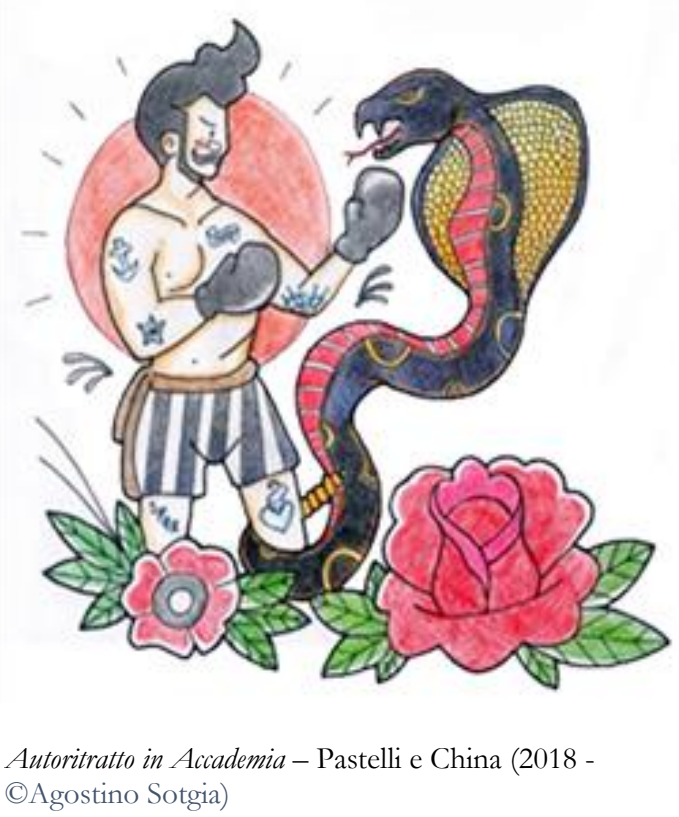


Martina Revello Lami: Ciao Agostino e grazie ancora per aver non solo deciso di condividere la tua creatività sul nostro giornale, ma soprattutto per aver ideato dal nulla un'immagine originale per questo numero di Ex Novo. Allora, come si evince dalle poche righe introduttive, professionalmente sei un archeologo e un ricercatore, in questa occasione prestato allillustrazione con un chiaro riferimento alla grafica fumettistica. Ci puoi raccontare come inizia questa passione? Tutti abbiamo un passato da voraci lettori di fumetti, ma da qui a fare il salto a disegnarne c'è un bel po'di strada....

Agostino Sotgia: Per rispondere a questa domanda procedo a ritroso e parto dalla fine perché il salto in realtà non l'ho fatto: ho certo un passato e un presente da lettore di fumetti, ma non ne disegno (ancora!). Il fumetto ha tutta una serie di regole e di linguaggi a cui mi sto approcciando solo ora, e il disegno ne è solo una delle tante componenti. Tuttavia, possiamo dire che al disegno, invece, io $\mathrm{mi}$ sono avvicinato prestissimo, avendo entrambi i genitori architetti sono cresciuto in mezzo a matite, pennini, scalimetri, colori, per cui mi è venuto naturale ad un certo punto avere "la matita in mano". Disegnare per me è stata una sorta di imprinting. Papà aveva un quadernetto sempre con sé, su cui disegnava i suoi schizzi del mondo circostante, delle persone che incontrava, o delle

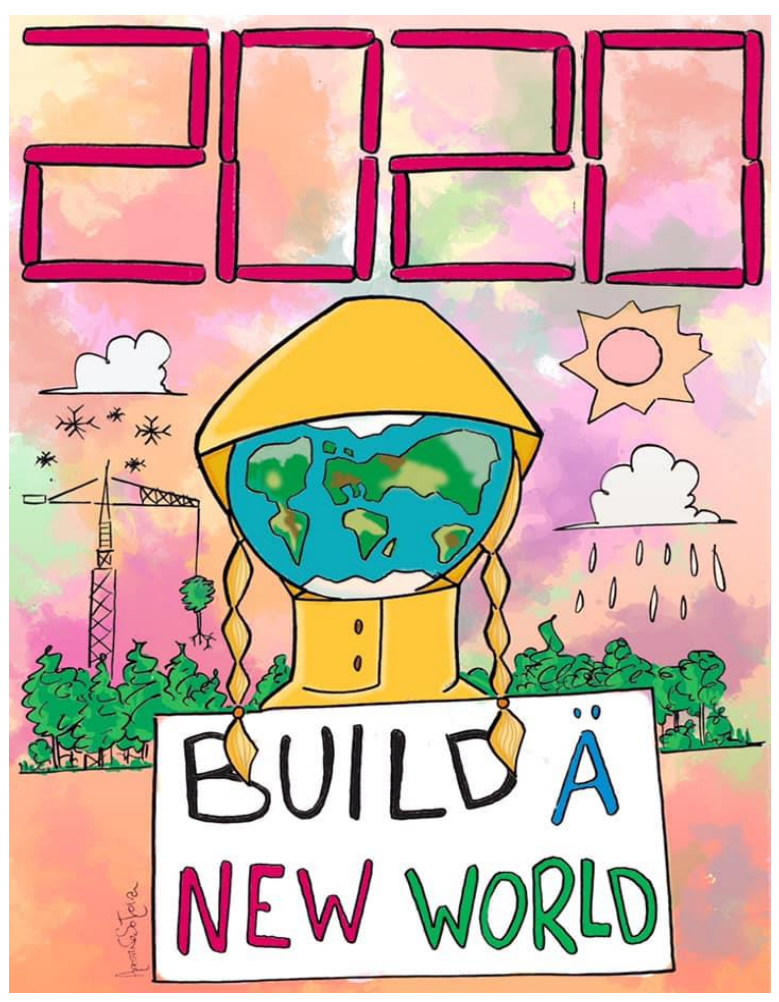

Happy New Year - China e Pittura Digitale (2019 - CAgostino Sotgia) situazioni in cui si trovava e mi diceva sempre: "Se non disegni una cosa la guardi ma non la osservi, non la capisci veramente né la ricordi”. Disegnare è diventato quindi il mio veicolo per osservare il mondo e per provare a raccontarlo anche agli altri.

I miei disegni sono una sorta di appunti, molto personali, di quello che mi circonda a metà tra un diario di viaggio e un Cabiers de doléances.

Venendo invece al fumetto, come ti dicevo, ci sono anche altre componenti fondamentali che lo rendono un mezzo d'espressione molto più trasversale e interessante ... rispetto ai miei lavori auto-analitici e un po' auto-referenziali.

A parte il disegno ci sono le inquadrature che determinano la carica narrativa del lavoro e poi ovviamente il racconto, la storia narrata dentro quei piani sequenza. Il fumetto è arte sequenziale, un po' come quando monti un video e scegli quali momenti della storia che stai narrando vuoi mettere in evidenza e soprattutto in che modo. Per esempio, nella copertina che abbiamo fatto ho deciso di mettere in primo piano il signore anziano di spalle (l'umarell, un classico nell'esperienza di cantiere di ogni archeologo) che osserva i lavori oltre la rete. In questo modo ho dato più risalto all'osservatore (con cui ci si dovrebbe immedesimare), e soprattutto alla barriera tra pubblico e addetti ai lavori. 
Ovviamente se questa copertina diventasse una storia a fumetti sarebbero seguite altre inquadrature, forse un primo piano dell'archeologa che vede con la coda dell'occhio lo spettatore e sa che a breve arriverà la fatidica domanda "Cosa state trovando?" e fa di tutto per evitare di incontrare il suo sguardo. Insomma, il fumetto è una forma di racconto molto più complessa dell'immagine statica di copertina, e non ti nascondo che ho la curiosità di provare a unire l'archeologia a questa forma di racconto, come già hanno fatto in Italia Emiliano Barletta e Alessio LoManto, di cui se non sbaglio Ex Novo ha già pubblicato un lavoro.

Martina Revello Lami: Quindi infanzia passata disegnando tra taccuini, pennelli e matite e anche leggendo fumetti, a cui poi si affianca la tua formazione come archeologo. Io ricordo la mia sorpresa quando appena iscritta all'università compresi l'importanza dello strumento grafico in archeologia, quanto disegnare fosse parte integrante della documentazione e comprensione del record archeologico. Nel tuo percorso, la consapevolezza che disegnare sia una componente essenziale di questa professione ti ha fatto cambiare approccio all'atto creativo? Insomma, disegnare per lavoro - forse un po'meccanicamente - che impatto ha avuto sulla tua passione per l'illustrazione? Ti ha avvicinato o allontanato dall'atto creativo?

Agostino Sotgia: Questa è una bella domanda... Diciamo che io ho un rapporto ambivalente in realtà con il disegno se diviene strumento di lavoro. Quando ho iniziato il mio percorso di studio il fatto di saper tenere in mano la matita mi ha aiutato, sono infatti stato subito reclutato sia per realizzare la documentazione grafica dei materiali e di scavo. Devo dire che non mi è mai pesato passare interminabili giornate in magazzino (purché ci fosse una fonte di caffè nelle vicinanze) a disegnare cocci o sotto il sole a documentare muri, evitavo così di stare al piccone, quindi l'ho sempre fatto molto volentieri. Tuttavia, il mio rapporto con il rilievo archeologico è ambivalente, perché mancando la parte più creativa è di fatto un'azione ben diversa dal disegnare un'illustrazione, possiamo dire anche più noiosa. Le norme da seguire

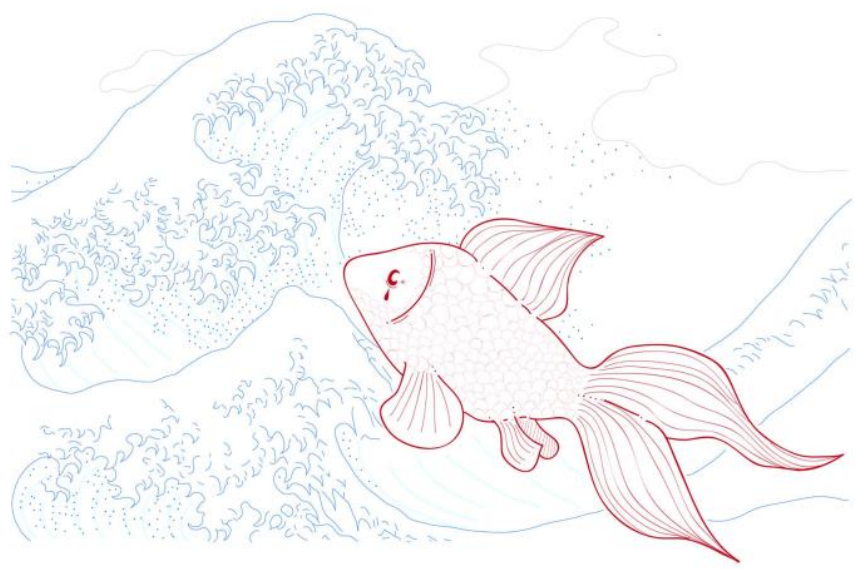

Da grande sarò una Carpa-Koi - Grafica Vettoriale (2017 - (C)Agostino Sotgia)

di "barare" un pochino, per esempio con le ombregoiature - che un tempo erano molto più usate nella rappresentazione grafica dei materiali - in quel caso l'abilità dell'illustratore 
riesce a rendere molto più realisticamente l'oggetto rappresentato anche se tutte le norme assonometriche non sono state applicate in pieno. Oggi siamo decisamente più abituati ad illustrazioni digitali, che non solo correggono i possibili difetti del disegno originale, ma permettono una standardizzazione molto maggiore e quindi un facilità nella lettura e comparazione degli oggetti.

In sostanza il bel disegno non è una necessità nella documentazione scientifica, ti dà una marcia in più e rende sicuramente il lavoro molto meno noioso, ma non è assolutamente lo scopo del "disegno archeologico", anzi molte volte è fuorviante.

Martina Revello Lami: Ma quindi tutte le ore passate nei magazzini freddi a disegnare cocci ti hanno allontanato o avvicinato al disegno?

Agostino Sotgia: Dipende, c'è tutta una componente ripetitiva e tecnica nel rilievo archeologico che assomiglia al solfeggio, va fatta ma certo rende disegnare assai più monotono. Allo stesso tempo, proprio grazie a questa ripetizione e alla pratica continua non ho mai perso il contatto con tutte quelle tecniche e norme che ci fanno costruire immagini realistiche e geometricamente corrette, una capacità che oggi posso sfruttare nel mio percorso di avvicinamento al fumetto, se pensiamo ad esempio alla costruzione della prospettiva o alla "teoria delle ombre". Insomma, le due componenti, creativa e normativa, che ho appreso e assorbito in maniera molto differente nel corso della mia formazione hanno una relazione reciproca e si compensano a vicenda.

Però se devo scegliere tra disegnare per ore tazze-carenate o Spider-Man ... la scelta ricade senza dubbio sull'arrampicamuri.

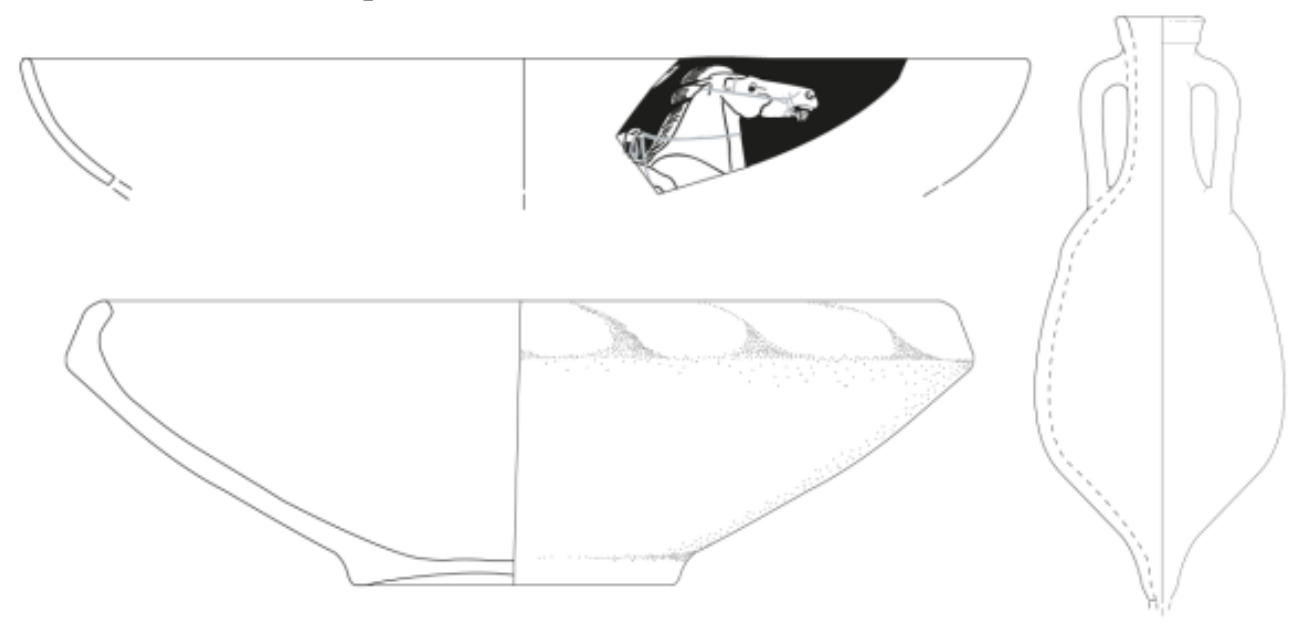

CAgostino Sotgia

Martina Revello Lami: Passiamo alla copertina di Ex Novo. L'immagine che hai creato parla non solo della tua passione per l'illustrazione, ma anche della tua esperienza sui cantieri urbani come archeologo. Immagino in molte occasioni ti sarai trovato nella situazione disegnata sulla copertina: circondato da una rete di protezione, in mezzo a ruspe, pale e segnaletica stradale, scrutato da passanti più o meno incuriositi dal movimento della terra. Quale è stato il tuo rapporto con il pubblico in quelle occasioni? $\mathrm{Ci}$ sono state occasioni in cui hai dovuto interagire e spiegare il tuo lavoro o rispondere alla fatidica domanda "Ma state trovando qualcosa"? 
Agostino Sotgia: $\mathrm{Si}$ esatto, nel mio percorso ci sono anche i cantieri urbani, croce e delizia del nostro lavoro. È proprio lì infatti che gli archeologi si trovano spesso per la prima volta a contatto con le altre parti coinvolte nella gestione/fruizione del bene culturale, vale a dire la committenza e il pubblico. Purtroppo, la formazione universitaria non ti prepara a gestire questo tipo di rapporti e ad aggravare le cose si aggiunge anche una certa difficoltà a riconoscere un ruolo istituzionale

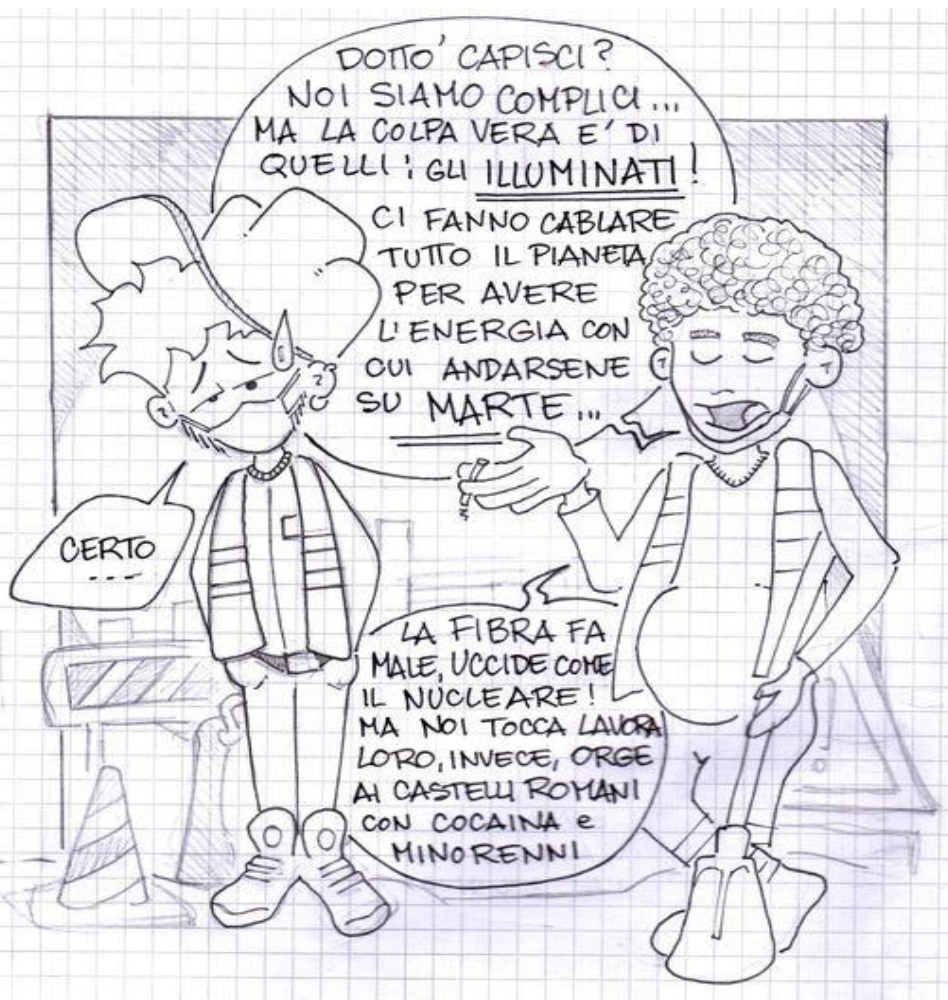
all'archeologo in cantiere,

Dottò ... - Matita e pennarello su foglio a quadretti (2020 - CAgostino Sotgia) per cui risulta difficile farsi accettare come una figura necessaria alla realizzazione del progetto in corso, piccolo o grande che sia. Non solo, l'archeologo diventa spesso il parafulmine, perché il lavoro di supervisione ti fa stare ore a bordo scavo ad osservare il movimento terra, quindi, sembri meno indaffarato e diventi automaticamente il filtro tra addetti ai lavori e passanti. Devo dire che nella mia personale esperienza mi è capitato di tutto: gente arrabbiata, insofferente, spesso a ragione perché per esempio la committenza cambia e lo stesso scavo viene riaperto più volte da ditte diverse e i lavori si allungano inspiegabilmente. Quindi è lecito chiedere e soprattutto è comprensibile un certo grado di insofferenza di fronte all'ennesimo intoppo alla circolazione. $\mathrm{E}$ in genere uno dei commenti più comuni che viene rivolto all'archeologo è "...ma di sicuro non cé niente, e se c'era hanno già scavato tutto!", dettato chiaramente dalla frustrazione di non capire cosa stia bloccando la viabilità.

Questo deriva anche dal fatto che l'archeologia urbana non è visibile: materialmente reti e recinti invalicabili non rendono comprensibile cosa avviene nel cantiere, il che rende qualunque tipo di comunicazione con il pubblico ancora più impervia. Nella copertina del volume ho rappresentato una versione molto "soft" di questa barriera: una transenna leggera in ferro (Orsogrill) dalla quale il nostro protagonista, il passante, può sbirciare dentro il cantiere e vedere sia le macchine che l'archeologa all'opera. In realtà nei cantieri urbani dove si scende oltre i $70 \mathrm{~cm}$, è assai raro l'utilizzo di questo tipo di recinzione: la committenza in genere opta per veri e propri muraglioni che impediscono del tutto la visione del sito. Ovviamente la mia scelta è stata dettata da motivi narrativi, altrimenti la copertina sarebbe stata un uomo davanti ad un muro... Ma questa è la situazione in cui gran parte dei cittadini si trova nelle nostre città quando si apre uno scavo. E li si crea un cortocircuito, in cui quanto più gli addetti ai lavori si isolano dal pubblico tanto più la 
tutela del patrimonio archeologico viene percepita come un intoppo (e per estensione anche gli archeologi) allo sviluppo della città contemporanea. Nel disegno di copertina, comunque, seppure questa dialettica è accennata, c'è una nota ottimista, infatti per me l'osservatore al di là della recinzione, l'umarell, è una figura positiva perché dimostra ancora curiosità e interesse per la città o anche solo per il proprio quartiere che cambia. Ed è questa curiosità che contiene credo la cura per il cortocircuito di cui sopra: se impariamo a raccontare meglio come la modernità può convivere con l'antichità, forse possiamo modificare la percezione del patrimonio archeologico come appunto un intoppo.

Martina Revello Lami: Dunque il disegno di copertina illustra bene quanto sia difficile comunicare il patrimonio archeologico al pubblico, specialmente quando si lavora in emergenza e in condizioni spesso precarie sui cantieri in città. Da anni ormai discutiamo su questo tema, eppure ancora troppo spesso gli esempi virtuosi almeno in Italia sono pochi. Pensi che gli archeologi siano preparati adeguatamente e sufficientemente riconosciuti come i mediatori più appropriati per illustrare le evidenze archeologiche? $\mathrm{E}$ che ruolo potrebbero avere i fumetti o l'arte grafica in generale per accorciare le distanze tra specialisti e pubblico?

Agostino Sotgia: Adesso dirò un'altra cosa impopolare. Secondo me parte del problema è endogeno, nasce all'interno della disciplina archeologica ed ha a che fare con l'annosa dicotomia tra ricerca scientifica e ricerca sul campo, tra accademia e professione. Troppo spesso gli stessi archeologi vedono il lavoro di supervisione in cantiere come un piano B rispetto alla ricerca, il che ovviamente comporta una narrazione sbagliata e quindi una percezione altrettanto errata di ciò che siamo chiamati a fare. Secondo me dobbiamo rimettere al centro l'archeologia urbana non come ultima spiaggia, ma come una parte importante del nostro lavoro: gli archeologi che supervisionano l'alloggiamento dei cavi della fibra ottica o seguono per anni i lavori della metropolitana ricoprono un ruolo istituzionale, sono di fatto i delegati della Soprintendenza (quindi dello Stato), non certo ricercatori relegati ad un piano B. La divisione tra ricerca e lavoro su campo va affrontata molto più fortemente allinterno della disciplina archeologica stessa e in questo senso tanto è stato fatto dalle associazioni di categoria, come la CIA, ma dal mio modesto punto di vista decisamente rimane ancora molto da fare.

Ciò detto, poi bisogna pure capire come comunicare ai più i processi di formazione della città moderna e come essa si stratifica. Da archeologo sono preparato a spiegare come la città antica si forma, ma solo grazie al lavoro nei cantieri urbani ho compreso come si sviluppa quella moderna: come funziona il reticolo di gallerie sotto i nostri marciapiedi e strade, come si creano i servizi e le connessioni necessari al funzionamento della città, insomma a cosa servono gli innumerevoli scavi che giornalmente vediamo aprirsi nei nostri quartieri. Se ribaltiamo la copertina e ci mettiamo dall'altra parte della recinzione, al posto dell'archeologa, capiamo quanto il nostro punto di osservazione ci collochi tra coloro che forse meglio possono accorciare la distanza tra pubblico e tutela. 
Venendo più concretamente alle strategie di comunicazione del patrimonio archeologico e del nostro ruolo di tutela, ci sono molti esempi ed i migliori secondo me utilizzano proprio il mezzo visivo, il racconto illustrato, le immagini. E' innegabile, d'altronde, che ormai la comunicazione moderna avvenga quasi esclusivamente per immagini, tanto sui social quanto sugli altri media.

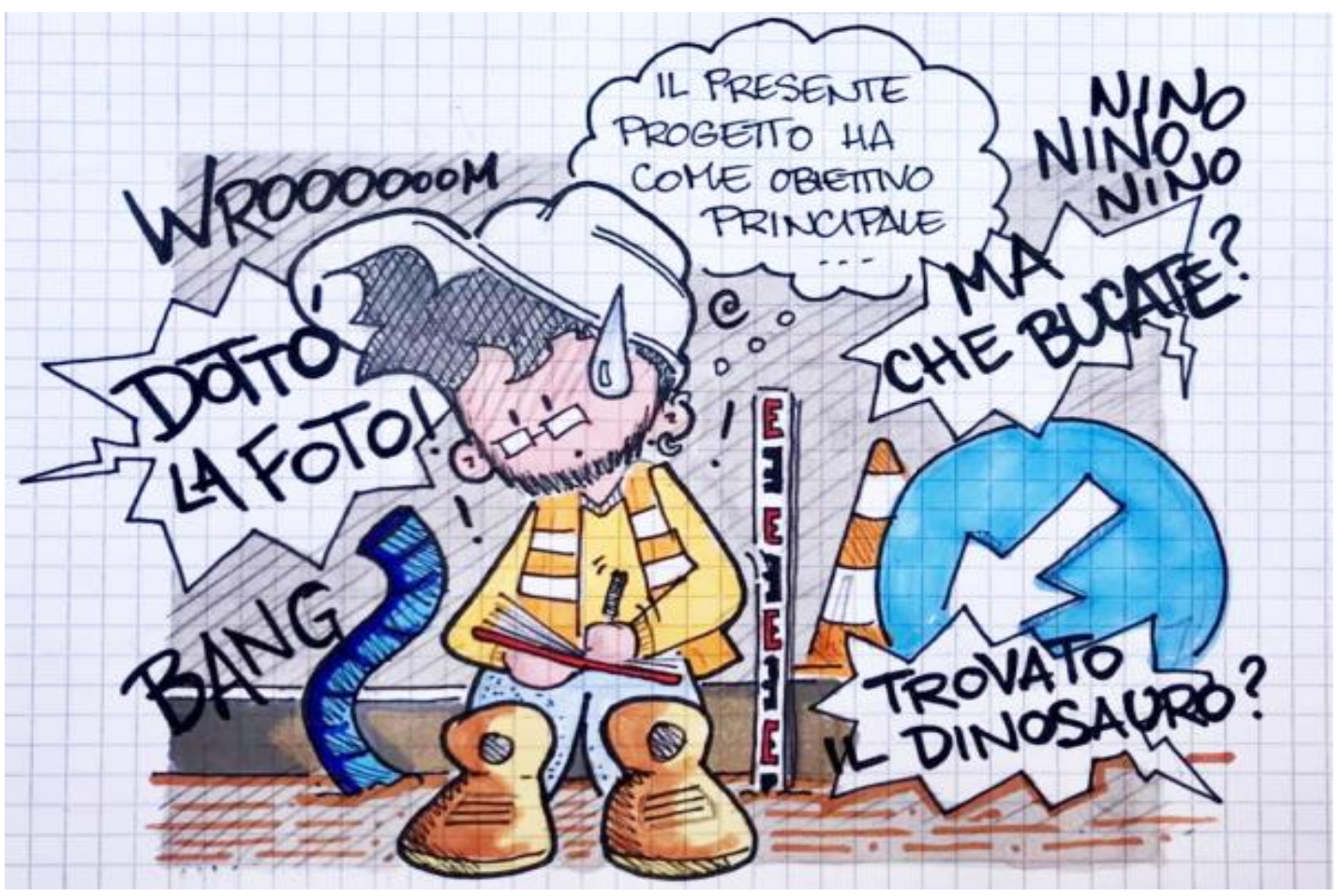

Torno Subito... - Pennarelli Pantone su foglio a quadretti (2019 - (C)Agostino Sotgia)

Tornando proprio ai fumetti, c'e stata un'importante mostra al Louvre sul tema e l'archeologia figurava come uno degli argomenti principali trattati in molte storie illustrate (L'archéologie en bulles - 26 settembre 2018-1 luglio 2019). Su suolo nazionale citerei ad esempio la rassegna Fumetti nei Musei in cui 51 musei sono stati presentati attraverso altrettanti albi con un buon riscontro di pubblico (https:// fumettineimusei.it/). Riguardo la professione dell'archeologo i lavori del già citato duo Barletta-Lo Manto, Archaeologye Comics pubblicato su Ex Novo, Un giorno di Ordinaria Archeologia curato insieme alla CIA e Diario di Scavo per Obló, hanno dato un contributo importante nel descrivere realisticamente la figura professionale dell'archeologo staccandola da narrative obsolete. Adesso credo i tempi siano maturi per fare il salto di qualità e tentare di raccontare da specialisti anche altre storie, non solo riguardanti il nostro ruolo nella società che come dicevo è stato già spiegato molto bene in diverse sedi. Abbiamo tutti gli strumenti oggi per divulgare storie più complesse in modo più coinvolgente o come direbbe Eco "popolare, comprensibile, trasversale e divertente". La narrativa legata a graphic novel e fumetti sta vivendo un momento di grandissimo successo nel nostro paese (pensiamo ad esempio alla serie firmata da Zerocalcare e prodotta da Netflix, o alla posizione dei suoi fumetti nelle classifiche negli ultimi dieci anni): come addetti alla tutela del patrimonio culturale non possiamo perdere questa occasione per essere molto più incisivi nella nostra comunicazione scientifica ed anche divulgativa. E soprattutto per non confinare i nostri studi alle mere antichità. 
Martina Revello Lami: Verissimo! Grazie Agostino per aver condiviso con noi non solo le tue abilità grafiche ma anche le tue riflessioni su archeologia urbana e divulgazione scientifica. Mi piace l'idea di non confinare i fumetti solo ad un tipo di comunicazione, ma di usarli trasversalmente...ovviamente Ex Novo è pronto ad ospitare altri progetti che vadano questa direzione!

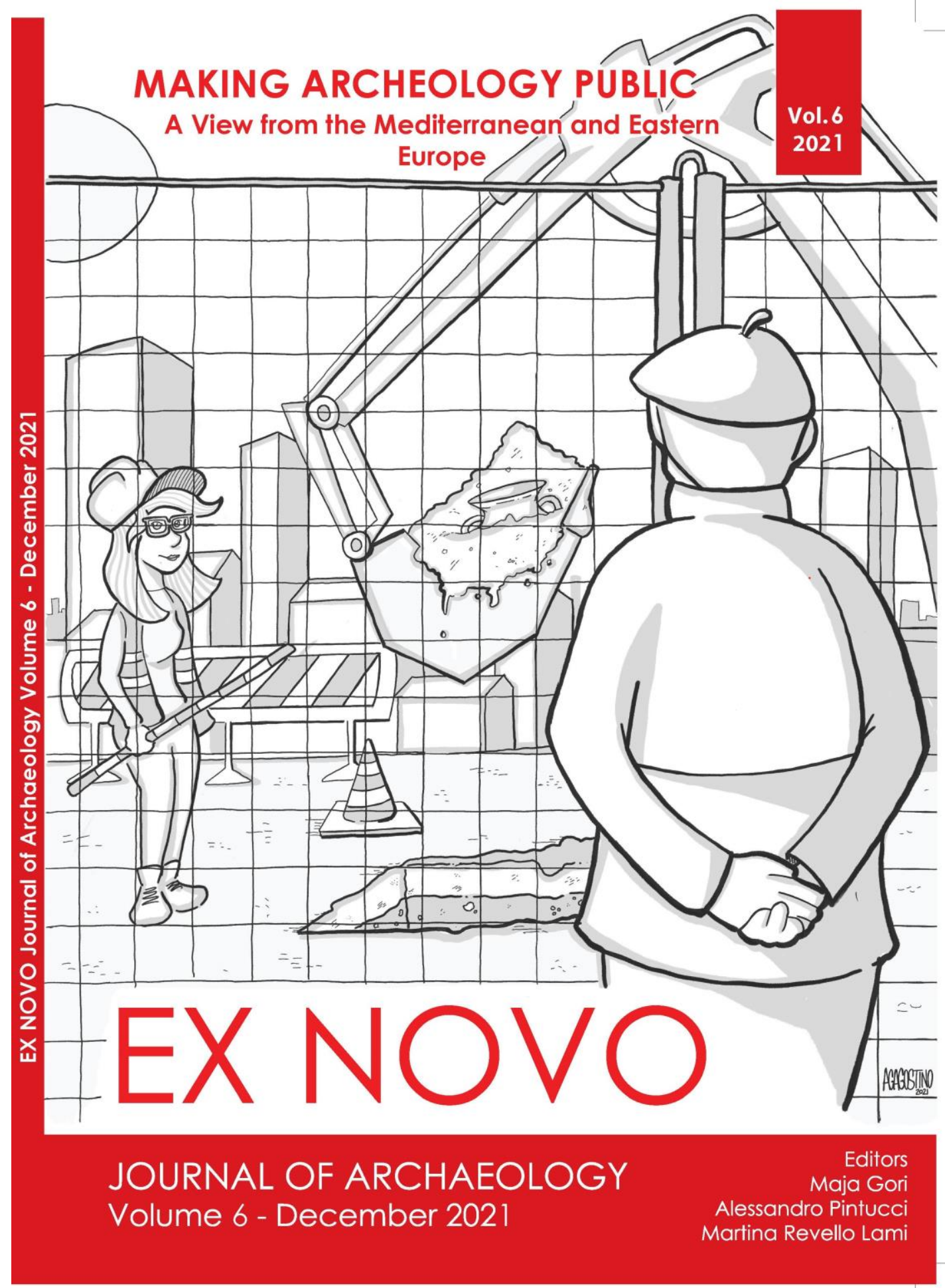

Something About Public Archaeology - Disegno Digitale (2021 - (CAgostino Sotgia) 